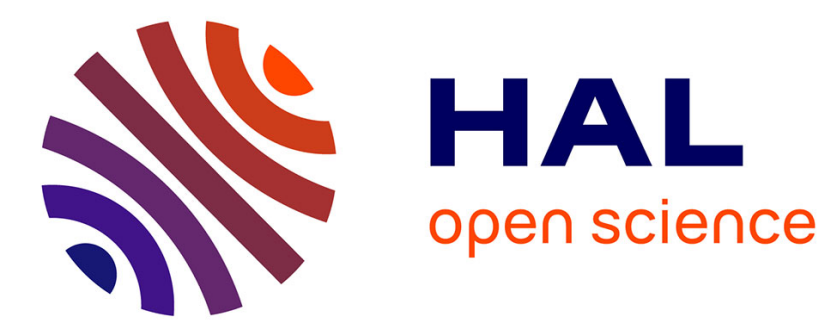

\title{
Water in oil microemulsions: criteria for dilution at constant droplet size
}

\author{
A.M. Cazabat
}

\section{To cite this version:}

A.M. Cazabat. Water in oil microemulsions: criteria for dilution at constant droplet size. Journal de Physique Lettres, 1983, 44 (14), pp.593-599. 10.1051/jphyslet:019830044014059300 . jpa-00232236

\section{HAL Id: jpa-00232236 https://hal.science/jpa-00232236}

Submitted on 1 Jan 1983

HAL is a multi-disciplinary open access archive for the deposit and dissemination of scientific research documents, whether they are published or not. The documents may come from teaching and research institutions in France or abroad, or from public or private research centers.
L'archive ouverte pluridisciplinaire HAL, est destinée au dépôt et à la diffusion de documents scientifiques de niveau recherche, publiés ou non, émanant des établissements d'enseignement et de recherche français ou étrangers, des laboratoires publics ou privés. 
Classification

Physics Abstracts

$78.20 \mathrm{~J}-78.35-82.70$

\title{
Water in oil microemulsions : criteria for dilution at constant droplet size
}

\author{
A. M. Cazabat
}

Laboratoire de Spectroscopie Hertzienne de l'E.N.S., 24, rue Lhomond, 75231 Paris Cedex 05, France

(Reçu le 6 avril 1983, accepté le 19 mai 1983)

\begin{abstract}
Résumé. - On discute la validité de la procédure de dilution des microémulsions utilisée dans de nombreux laboratoires, notamment aux concentrations élevées et au voisinage de points critiques de démixtion.
\end{abstract}

Abstract. - The validity of the customary method for diluting water in oil (w/o) microemulsions is discussed. In concentrated systems and in the vicinity of critical consolute points, the method has deficiencies.

\section{Introduction.}

At low water content, water in oil microemulsions are dispersions of identical spherical water droplets in a continuous oil phase stabilized by the presence of surfactant [1]. As droplets collide, transient aggregates of various sizes may form [2]. The probability of sticky collisions depends on the interactions between droplets.

Scattering studies [3] (X-rays, neutrons, light) allow measurement of the size of droplets and the interactions between them, provided a dilution procedure is available to vary the volume fraction $\phi$ of droplets without changing their size.

Such a procedure, first proposed by Schulman [4], has been improved by Graciaa [5] :

First, oil is added to a transparent microemulsion. The sample becomes milky. Transparency is obtained again by adding alcohol containing a certain amount of water. This is repeated several times and finally the quantity of added alcohol is plotted against the quantity of added oil. A linear plot indicates a constant composition of the added substances, which therefore constitute the microemulsion continuous phase. Because the composition of the dispersed phase has not been altered, it follows that if the microemulsion is still a dispersion of identical spherical droplets, then their size has been kept constant.

The linearity requirement leads to a very precise determination of the composition of oil, alcohol and water in the continuous phase : an improper amount of water in the added alcohol leads to obvious curvature in the dilute range $(\phi<0.05)$ [5].

By contrast, the method is not really sensitive to the composition of the dispersed phase, especially in the more concentrated range $(\phi>0.05)$. Moreover, it is necessary to suppose that the microemulsion structure is not changed by the dilution process. However, this hypothesis is not supported by scattering experiments in this concentration range $(\phi>0.05)$. 
As this method of dilution is widely used in French and Dutch laboratories, but is very much debated in some other countries [6], it seemed to us useful to discuss its validity.

\section{Concentrated microemulsions.}

An obvious limitation of the dilution method is easily observed in the concentrated range. In figure 1, the total amount of alcohol (and water) is plotted against the total amount of oil (droplets included) for several series of w/o microemulsions whose properties are listed in table I.

Table I. - w/o microemulsions systems.

\begin{tabular}{|l|c|c|c|c|c|}
\hline Code & Surfactant & Oil & Alcohol & $\begin{array}{c}\text { Droplet } \\
\text { radius } \\
\AA\end{array}$ & $\beta$ \\
\hline ATP & SDS & T & P & 45 & +7 \\
ACP & SDS & C & P & 49 & +7 \\
BCP & SDS & C & P & 81 & -1 \\
$\alpha$ TB & SDS & T & B & 36 & -12 \\
ATB & SDS & T & B & 41 & -20 \\
$\beta$ TTB & SDS & T & B & 60 & -40 \\
$\gamma$ TB & SDS & T & B & 90 & $<-40$ \\
\hline
\end{tabular}

SDS : sodium dodecylsulfate, $\mathrm{T}$ : toluene, $\mathrm{C}$ : cyclohexane, $\mathrm{P}:$ pentanol, $\mathrm{B}$ : butanol.

The right portions of the curves (dilute range) are linear, indicating that the composition of the continuous phase is constant. At higher droplet concentrations, curvature appears, indicating redistribution of the components. Redistribution allows the microemulsions to pass continuously to the inverted (oil in water) structure. The curvature is first visible in the series with large attractive interactions between droplets (as indicated by the second virial coefficients listed in table I), or with cyclohexane as oil. Looking at the linearity of the curves, it is obvious that constant size droplets cannot be obtained for $\phi>0.30$ in hard sphere systems, and $\phi>0.15,0.20$ in systems with large attractive interactions.

In this latter case, another type of limitation has to be taken into account in the vicinity of critical demixion points $[7,8]$.

\section{Critical demixion points.}

The size of microemulsion droplets and the nature of the interactions between them can be determined from static light scattering experiments.

The scattered intensity $I$ is related to the osmotic compressibility $\delta \pi / \delta \phi$ of the droplet dispersions through the formula [9] :

$$
I=K \phi\left(\frac{\partial n}{\partial \phi}\right)^{2}\left(\frac{\partial \pi}{\partial \phi}\right)^{-1}
$$

Here $K$ is a geometrical constant and $n$ the index of refraction of the microemulsion. 


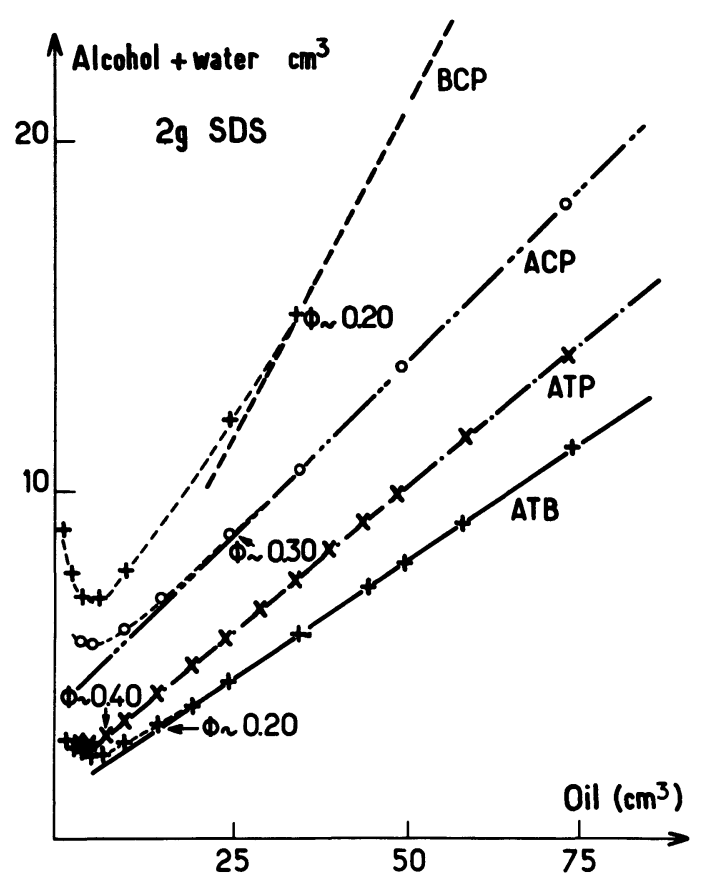

Fig. 1. - Total amount of alcohol plotted against total amount of oil (droplets included) for a series of microemulsions containing $2 \mathrm{~g}$ SDS. The volume fraction $\phi$ at the onset of the curvature is indicated.

At very low volume fraction $\phi$ :

$$
\pi \underset{\phi \rightarrow 0}{\longrightarrow} \frac{k T}{\gamma} \phi\left(1+\frac{\beta}{2} \phi\right)
$$

where $k$ is the Boltzmann constant, $T$ the absolute temperature, $\gamma$ the droplet volume, and $\beta$ a second virial coefficient defined by the relation 2 .

$$
\frac{\partial \pi}{\partial \phi} \underset{\phi \rightarrow 0}{\longrightarrow} \frac{k T}{\gamma}(1+\beta \phi)
$$

The normalized osmotic compressibility $\gamma / k T . \delta \pi / \delta \phi$ is plotted in figure 2 against the volume fraction $\phi$ for several series of microemulsions.

As the attractive potential $V$ between droplets increases, $\beta$ decreases. The curves pass closer and closer to the $\phi$-axis. Finally, for a critical value $V_{c}$, the $\delta \pi / \delta \phi$ curve becomes tangent to the $\phi$-axis. The point of contact $\phi=\phi_{c}$ is a critical demixion point.

The ATP series corresponds to $V \ll V_{c}$, the ATB series to $V<V_{c}$.

On the contrary, both the $\beta$ TB and the $\gamma$ TB series correspond to $V>V_{\mathrm{c}}$. In this case, keeping constant the droplet radius and increasing $\phi$ must lead to demixion. In practice, the samples are obtained by titration and no demixion is observed. The dilution curve bends a little [10], which is not easy to notice because of the titration difficulties (the samples are strongly opalescent), and goes through a critical point. At higher concentration, the curvature associated with the concentrated range becomes visible. On the whole, the curve looks fairly linear. Note however that the curve concavity in the high concentration range is changed. The curvature is positive for $V\left\langle V_{c}\right.$, negative for $V \gg V_{c}$. For $V>V_{c}$, discontinuous situations with lobes in the phase diagram are observed. These features are illustrated in the dilution curves shown in figure 3. 


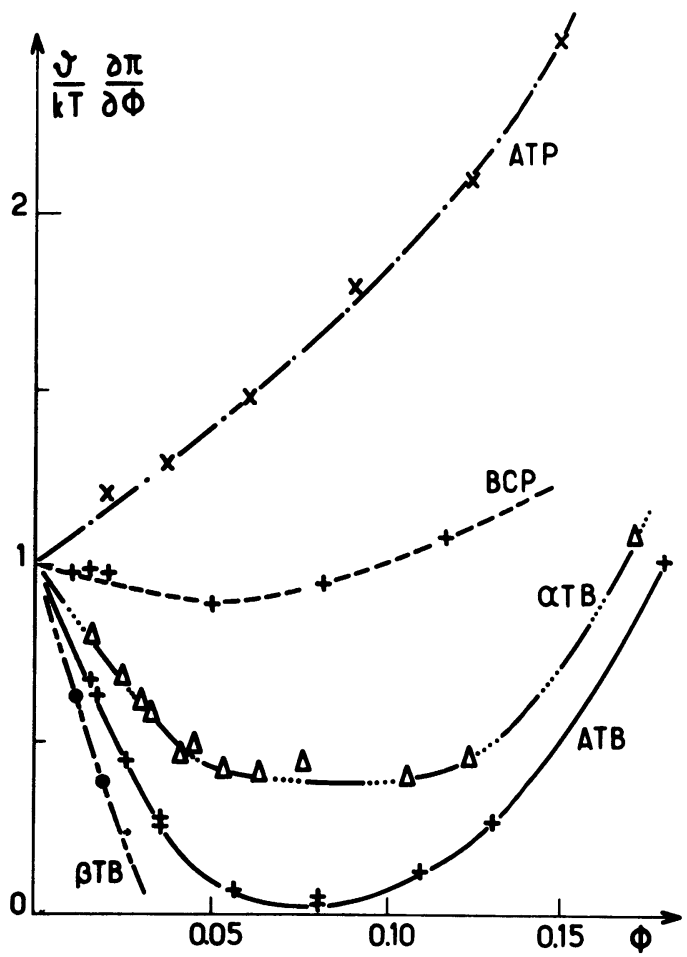

Fig. 2. - Reduced osmotic compressibility $\delta \pi / \delta \phi \cdot \gamma / k T$ plotted against volume fraction $\phi$ for several systems.

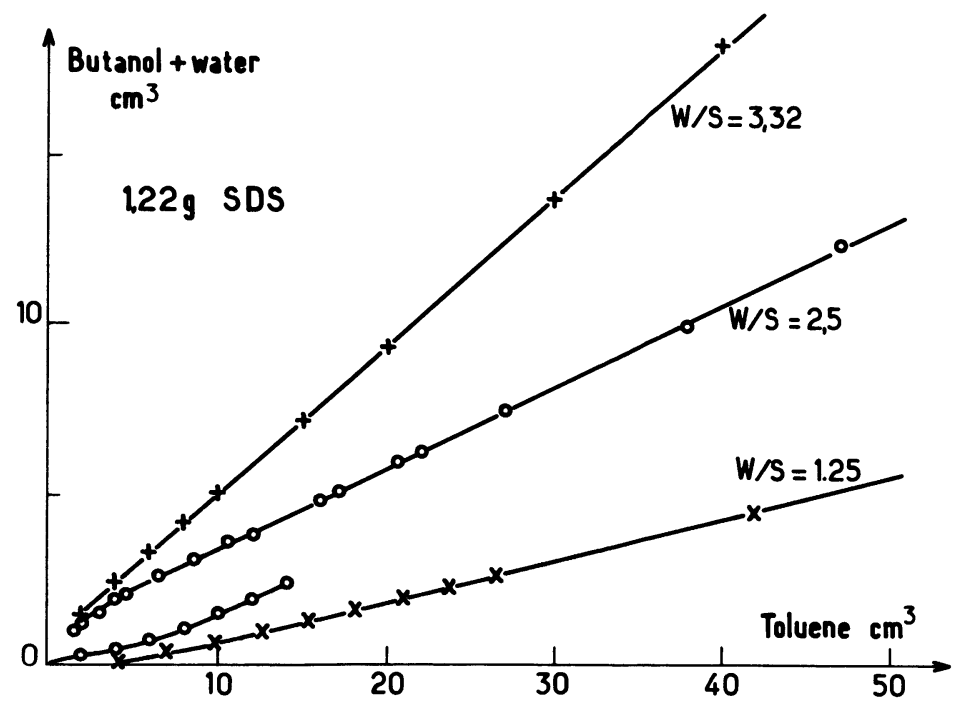

Fig. 3. - Dilution curves for systems containing 1.22 SDS W/S is the water to soap ratio by weight in the droplets. The larger $\mathrm{W} / \mathrm{S}$, the larger the interaction potential. The critical interaction potential $V_{\mathrm{c}}$ is obtained for $\mathrm{W} / \mathrm{S}=1.5 ; \mathrm{W} / \mathrm{S}<1.5, V<V_{\mathrm{c}} ; \mathrm{W} / \mathrm{S}>1.5, V>V_{\mathrm{c}}$. 
Similar behaviour is observed for cyclohexane-butanol systems.

It follows from the preceding analysis that in $V>V_{\mathrm{c}}$ microemulsions, droplets cannot keep a constant size during dilution, except at very low concentrations $\phi<0.02$. In spite of this, the dilution curves can look linear.

Evidently, a plot of alcohol against oil content at constant soap content is not sensitive enough to the composition of the dispersed phase. However if alcohol content is plotted versus soap content at constant oil content (systems with increasing $\phi$ ), no such ambiguity is observed. Only $V<V_{\text {c }}$ curves look linear.

The changing structure of the dispersed phase in $V>V_{\mathrm{c}}$ microemulsions can easily be observed using transient electric birefringence or electrical conductivity measurements.

In figure 4 , the Kerr constant $B$ is plotted against $\phi$ for several microemulsion series.

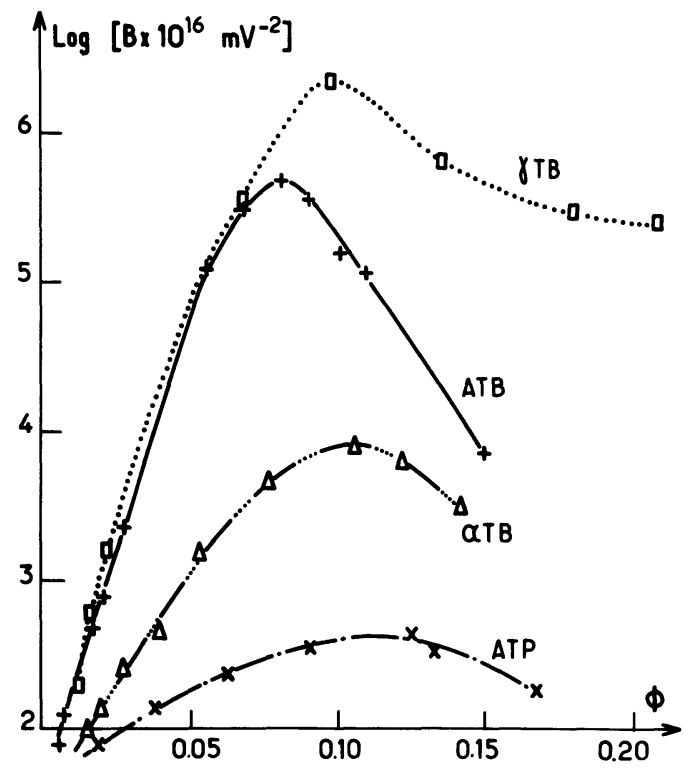

Fig. 4. - Plot of the logarithmic Kerr constant $B$ versus volume fraction $\phi$ for several microemulsion systems.

In the intermediate concentration range $\phi<15 \%$ the Kerr constant is related to concentration fluctuations.

Very high Kerr constants are measured in the vicinity of critical points. However, a distinct feature of the $\gamma$ TB system is the very slow decrease of $B$ for $\phi>10 \%$. The turbidities of the samples also decrease very slowly in this concentration range. The $\gamma \mathrm{TB}$ curve is not in agreement with a model of constant size droplets, which would predict a more symmetrical curve, like that of the ATB system.

In figure 5, the electrical conductivity $K$ is plotted against the volume fraction $\psi$ of water for the same systems. As the continuous phase is non-conducting, the conductivity probes the connections between droplets.

A qualitative explanation of the curves is the following :

- For $V \ll V_{\mathrm{c}}$, the microemulsion droplets are randomly distributed. An infinite droplet path arises for $\phi>\phi_{\mathrm{g}} \sim 14 \%$ (geometrical percolation [11] or site percolation). The probability of connection between neighbouring droplets depends on the attractive potential $V$ [12]. Large values of $K$ will be observed for $\phi>\phi_{\mathrm{p}}$ (site-bond percolation). The larger $V$ is, the closer will be $\phi_{\mathrm{p}}$ and $\phi_{\mathrm{g}}[13,14]$. 


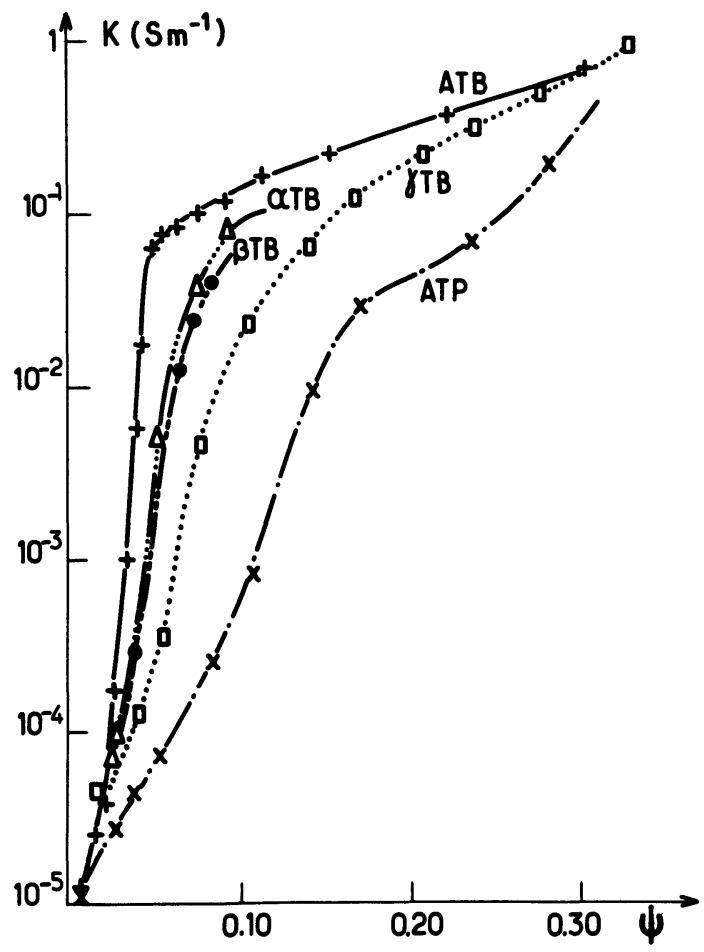

Fig. 5. - Plot of the logarithmic electric conductivity $K$ versus the water volume fraction $\psi$ for several systems.

- For $V \lesssim V_{c}$, the spatial distribution of droplets is modified by the attractive potential $V$. The medium is no longer homogeneous over a light wavelength and becomes opalescent.

In this case, $\phi_{\mathrm{g}}<14 \%$ and $\phi_{\mathrm{g}} \# \phi_{\mathrm{p}}$. - The electrical conductivity increases very steeply, reflecting percolation of the conductivity [13].

- For $V>V_{\mathrm{c}}$ both the structure of the dispersed phase and $V$ itself change along the dilution line : this can explain the more gradual increase of the conductivity curves.

To summarize, a valid dilution procedure is only possible if :

$$
V<V_{\mathrm{c}} \quad(\beta>-20)
$$

and $\phi<20 \%$.

Satisfying these two criteria ensures a constant composition of the dispersed phase.

What is the structure of this phase ? Problems will arise in the case of very frequent sticky collisions with merging and breaking up of transient droplets. In this case, the structure of the medium, averaged over some microseconds (for light scattering experiments), could be amoebic, not a dispersion of individual droplets.

Clearly, this process is bound to occur above a given concentration for $V<V_{c}$. However, neutron and light scattering experiments [3] do not seem to indicate the process to be more problematical than the issue of constant composition of the dispersed phase. This can be explained by considering the Talmon and Prager model for microemulsion structure [15]. In this model no interactions exist and the droplets are randomly distributed, except that the probability of sticky collisions is one. This clearly leads to predicting the existence of complicated structures 
even at low volume fractions. In fact, however, collision transfer studies [16] in microemulsions have found very small values for this probability, on the order of $10^{-3}$. Even in attractive systems, the values are probably much less than unity.

Hence the predicted structural complexity of the Talmon and Prager model is probably a strong overestimate compared to reality.

Finally, we recall the criteria of a valid dilution procedure :

$$
\begin{array}{lll}
V<V_{\mathrm{c}} & \beta>-20 \\
\phi<\phi_{\max } & \phi_{\max } \sim 0.3 \text { for } V \ll V_{\mathrm{c}} \\
& \phi_{\max } \sim 0.15 \text { for } V<V_{\mathrm{c}} .
\end{array}
$$

Within these bounds, the concentration of droplets can be varied while keeping their size constant, and quantitative determinations concerning droplet size and the interactions between them can be made.

\section{References}

[1] Schulman, J. H., Friend, J. A., Koll. Z. 115 (1949) 67.

[2] Eicke, H. F., Shepherd, J. C. W., Steineman, A., J. Colloid Interface Sci. 56 (1976) 168.

[3] a) Calje, A. A., Atgerof, W. G. M., VRIJ, A., in Micellization, Solubilization and Microemulsions, vol. 2 (Plenum Press) 1976.

b) Graclaa, A., Lachaise, J., Chabrat, P., Letamendia, L., Rouch, J., Vaucamps, C., J. Physique-Lett. 39 (1978) L-235.

c) Dvolaitzky, M., Guyot, M., Lagües, M., Lepesant, J. P., Ober, R., Sauterey, C., Taupin, C., J. Chem. Phys. 69 (1978) 3279.

d) Cazabat, A. M., Langevin, D., Pouchelon, A., J. Colloid Interface Sci. 73 (1980) 1.

Many other papers have been published recently.

[4] Schulman, J. H., Stoeckenius, W., Prince, L., J. Phys. Chem. 63 (1959) 1677.

[5] GraciaA, A., Thesis, Université de Pau (1978).

[6] This procedure disagrees with the hypotheses of the Talmon and Prager model [15].

[7] BourBon, D., Thèse de Docteur Ingénieur, Bordeaux 1981 and other papers of the Centre de Recherche Paul Pascal (P. Bothorel).

[8] Cazabat, A. M., Langevin, D., Meunier, J., Pouchelon, A., J. Physique-Lett. 43 (1982) L-89.

[9] Cazabat, A. M., Langevin, D., J. Chem. Phys. 74 (1981) 3148.

[10] Roux, D., BellocQ, A. M., Leblanc, M. S., to be published.

[11] Coniglio, A., Klein, W., J. Phys. A 13 (1980) 2775.

[12] Coniglio, A., Stanley, H. E., Klein, W., Phys. Rev. Lett. 42 (1979) 518.

[13] Lagües, M., Ober, R., Taupin, C., J. Physique-Lett. 39 (1978) L-487.

[14] Cazabat, A. M., Chatenay, D., Langevin, D., Pouchelon, A., J. Physique-Lett. 41 (1980) L-441.

[15] Talmon, Y., Prager, S., J. Chem. Phys. 69 (1978) 2984.

[16] Robinson, B. H., Steytler, D. C., TACK, R. D., J. Chem. Soc. Faraday Trans. I 75 (1979) 781. 